This item was submitted to Loughborough's Research Repository by the author.

Items in Figshare are protected by copyright, with all rights reserved, unless otherwise indicated.

\title{
Indoor UAV control using multi-camera visual feedback
}

PLEASE CITE THE PUBLISHED VERSION

http://dx.doi.org/10.1007/s10846-010-9506-8

\section{PUBLISHER}

(C) Springer Science+Business Media B.V.

\section{VERSION}

AM (Accepted Manuscript)

\section{PUBLISHER STATEMENT}

This work is made available according to the conditions of the Creative Commons Attribution-NonCommercialNoDerivatives 4.0 International (CC BY-NC-ND 4.0) licence. Full details of this licence are available at: https://creativecommons.org/licenses/by-nc-nd/4.0/

\section{LICENCE}

CC BY-NC-ND 4.0

\section{REPOSITORY RECORD}

Oh, Hyondong, Dae-Yeon Won, Sung-Sik Huh, David H. Shim, Min-Jea Tahk, and Antonios Tsourdos. 2015. "Indoor UAV Control Using Multi-camera Visual Feedback". figshare. https://hdl.handle.net/2134/17856. 


\title{
Indoor UAV Control Using Multi-camera Visual Feedback
}

Hyondong Oh*, Dae-Yeon Won, Sung-Sik Huh, David Hyunchul Shim and MinJea Tahk**, Antonios Tsourdos*

*Dept. of Informatics and Systems Engineering, Cranfield University, Swindon, UK

**Dept. of Aerospace Engineering, KAIST, Daejeon, South Korea

h.oh@cranfield.ac.uk, dywon@fdcl.kaist.ac.kr, sshuh@fdcl.kaist.ac.kr, hcshim@kaist.ac.kr, mjtahk@fdcl.kaist.ac.kr, a.tsourdos@cranfield.ac.uk

\begin{abstract}
This paper presents the control of an indoor unmanned aerial vehicle (UAV) using multi-camera visual feedback. For the autonomous flight of the indoor UAV, instead of using onboard sensor information, visual feedback concept is employed by the development of an indoor flight test-bed. The indoor test-bed consists of four major components: the multi-camera system, ground computer, onboard color marker set, and quad-rotor UAV. Since the onboard markers are attached to the pre-defined location, position and attitude of the UAV can be estimated by marker detection algorithm and triangulation method. Additionally, this study introduces a filter algorithm to obtain the full 6-degree of freedom (DOF) pose estimation including velocities and angular rates. The filter algorithm also enhances the performance of the vision system by making up for the weakness of low cost cameras such as poor resolution and large noise. Moreover, for the pose estimation of multiple vehicles, data association algorithm using the geometric relation between cameras is proposed in this paper. The control system is designed based on the classical proportional-integral-derivative (PID) control, which uses the position, velocity and attitude from the vision system and the angular rate from the rate gyro sensor. This paper concludes with both ground and flight test results illustrating the performance and properties of the proposed indoor flight test-bed and the control system using the multi-camera visual feedback.
\end{abstract}

Keywords Extended Kalman filter, Indoor flight test-bed, Multi-camera system, Marker detection, Quad-rotor UAV, Visual feedback 


\section{Introduction}

There has been a growing interest in a small unmanned aerial vehicle (UAV) in both civilian and military applications such as surveillance, reconnaissance, target tracking and data acquisition. Since it is difficult to accurately describe the aerodynamics of the small UAV, the verification of its performance by flight test plays an important role in developing the controller of the new vehicle. Most autonomous flight test was performed outdoor so that the reliable navigation system like the global positioning system (GPS) can be used. However, outdoor test-bed requires not only wide area, suitable transportation and qualified personnel but also tends to be vulnerable to the adverse weather condition. Accordingly, an indoor flight test-bed using a vision system is emerging as a possible solution recently. The indoor test-bed enables flight test which ensures protection from the environmental condition. In addition, vision system can provide accurate navigation information or be fused with other on-board sensors like GPS or inertial navigation system (INS) to bound error growth.

In this context, much progress has been made in control of an indoor aerial vehicle using vision system. The RAVEN (Real-time indoor Autonomous Vehicle test ENvironment) system developed by MIT ACL (Aerospace Control Lab) [1] estimates the information of the UAV by measuring the position of the maker installed in the UAV via beacon sensor used in motion capture. Although this system has a high resolution of $1 \mathrm{~mm}$ and can handle multiple UAVs, on the contrary, it has the disadvantage of requiring expensive equipment. Also, the twocamera pose estimation of the quad-rotor using a pair of ground and on-board cameras has been introduced [2]. Two cameras are set to face each other so that the full 6-degrees-of-freedom (DOF) pose of the UAV can be estimated. This system can be implemented with low cost, but it requires camera installation both indoor and on the UAV causing difficulty in testing multiple UAVs simultaneously. In [3], a visual control system for a micro helicopter has been developed. Two stationary and upward-looking cameras placed on the ground track four black balls attached to the helicopter. The errors between the positions of the tracked balls and pre-specified references are used to compute the visual feedback control input. Mak et al. [4] proposed a localization system for an indoor rotary-wing MAV that uses three onboard LEDs and base station mounted active vision unit. A USB webcam tracks the ellipse formed by cyan LEDs and estimates 
the pose of the MAV in real time by analyzing images taken using an active vision unit.

As aforementioned, a major challenge of vision system is to develop both lowcost and robust system which provides sufficient information for the autonomous flight, even for multiple UAVs. Moreover, previous researches have the limitation of providing only stationary information such as position and attitude. In other words, they cannot be applied alone to the control of the vehicle without other sensors. Thus, this paper introduced the filter algorithm to obtain the full 6-DOF pose estimation including velocities and angular rates. Filter algorithm can also enhance the performance of the test-bed system by making up for a weakness of low cost camera such as poor resolution and large noise. In addition, for the pose estimation of multiple vehicles, data association using geometric relation between cameras is proposed in this study.

The objective of this paper is the control of the indoor UAV utilizing only low cost cameras installed indoor. The quad-rotor UAV is considered as a platform vehicle since it has simple dynamics and can effectively operate in narrow indoor environments. Multi-camera visual feedback information for the quad-rotor UAV control is the full 6-DOF pose estimation and it is obtained from the indoor flight test-bed by using the vision algorithm and extended Kalman filter (EKF). Designing filter, the dynamic model of the quad-rotor UAV is the 6-DOF nonlinear equations and the measurements are the visual information of the color markers attached to the UAV which is obtained periodically from camera. Since there is a time delay between the actual color marker motion and its image data, modified EKF algorithm considering the delayed measurement is used. The control system is designed based on the classical proportional-Integral-Derivative (PID) control, which uses the visual feedback information of the position, velocity and attitude from the EKF and angular rate from the rate sensor. This paper is organized as follows: an overview of the structure of the indoor flight test-bed for visual feedback information and the operation concept is provided in section 2 , followed by the vision algorithm composed of the camera calibration and detection of the marker attached to the quad-rotor UAV. Next, dynamic model of the vehicle and measurement model of the camera is introduced and the EKF algorithm is explained. Section 5 illustrates the controller design for the quad- 
rotor UAV and section 6 shows experimental results of the proposed control system using multi-camera visual feedback.

\section{Problem Statement}

\subsection{Indoor Flight Test-bed}

For the autonomous flight of the indoor UAV, visual feedback concept is employed by the development of an indoor flight test-bed using multi-camera system. As shown in figure 2.1, our test-bed consists of four major components: the multi-camera system, ground computer, onboard color markers, and UAV. Designing the indoor test-bed, the number of camera and marker is an important factor. In most cases, when three markers from one camera are detected, the position and attitude of the vehicle can be determined by checking the rank of error covariance or Fisher information matrix. In case that there is a sign ambiguity or the endpoints of the marker position vectors are connected by a straight line, four or more markers are required [5]. Besides, as the number of camera and marker increases, the performance of the system, such as accuracy and robustness, is enhanced, however, the computation burden becomes heavier. In this study, the test-bed is composed of the two cameras and four known markers attached to the UAV so that the observability and reasonable performance can be guaranteed.

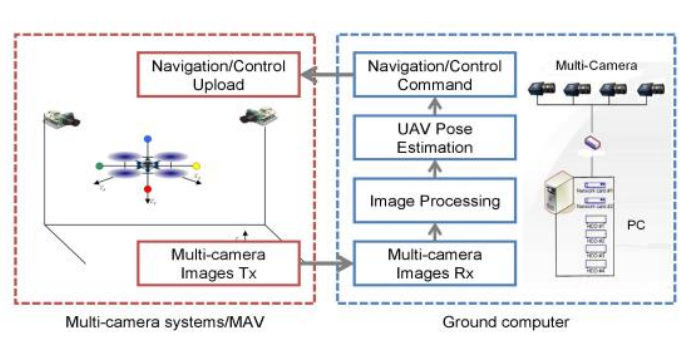

Figure 2.1 Indoor test-bed configuration

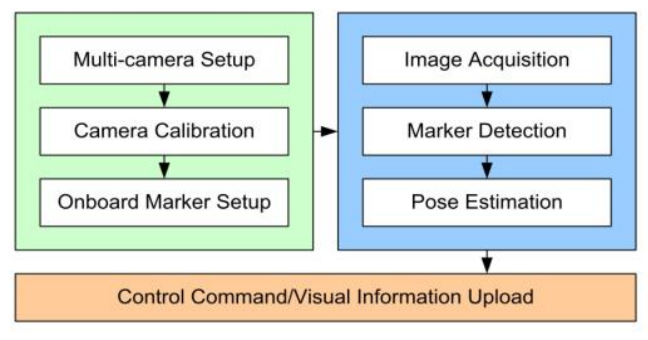

Figure 2.2 Operation procedure

\subsection{Operation Procedure}

The operation of the indoor flight test-bed starts from the multi-camera system setup. And the camera calibration to describe a mapping between the $3 \mathrm{D}$ world 
and a 2D image and the attaching of the onboard marker to the UAV are followed. When the flight test begins, the image of the entire environment including the UAV taken by the multi-camera system is transmitted into the ground computer. By analyzing obtained image, ground computer finds the position of the marker with respect to the camera image frame. Since the onboard markers are attached to the pre-defined position, the position and attitude of the UAV can be estimated by using marker position and filter algorithm with the dynamic and measurement model. The overall operation procedure is represented as shown Fig. 2.2.

\section{Vision Algorithm}

This section presents the vision algorithm for the pose estimation of the quadrotor UAV. First of all, camera model and calibration method are explained, and marker detection algorithm is presented. In addition, the concept of the multiUAV tracking is proposed.

\subsection{Camera Model and Calibration}

This paper considers the basic pinhole camera model designed for charge-coupled device (CCD) like sensor to describe a mapping between the $3 \mathrm{D}$ world and a $2 \mathrm{D}$ image. The basic pinhole camera model can be written as [6]:

$$
\overline{\mathbf{x}}_{\text {image }}=\mathbf{P} \overline{\mathbf{X}}_{\text {world }}
$$

where $\overline{\mathbf{X}}_{\text {world }}$ is the 3D world point represented by a homogeneous four element vector $\left(X, Y, Z, W_{s}\right)^{T}, \overline{\mathbf{x}}_{\text {image }}$ is the $2 \mathrm{D}$ image point represented by a homogeneous

vector $\left(x, y, w_{s}\right)^{T} . W_{s}$ and $w_{s}$ are the scale factors which represent the depth information and $\mathbf{P}$ is the 3 by 4 homogeneous camera projection matrix with 11degrees of freedom, which connects the 3D structure of the real world and 2D image points of the camera and given by:

$$
\mathbf{P}=K\left[R_{I}^{c a m} \mid t_{I}^{c a m}\right] \text {, where } K=\left[\begin{array}{ccc}
\alpha_{x} & s & x_{0} \\
0 & a_{y} & y_{0} \\
0 & 0 & 1
\end{array}\right]
$$


where $R_{I}^{\text {cam }}$ is the rotation transform matrix and $t_{I}^{\text {cam }}$ is the translation transform matrix from inertial frame to camera center frame and $\left(\alpha_{x}, \alpha_{y}\right),\left(x_{0}, y_{0}\right)$, s are the focal length of the camera in terms of pixel dimensions, principal point and skew parameter, respectively. Camera calibration procedure estimates the camera projection matrix which relates the 3D space and the corresponding image entries. In this study, camera projection matrices of multi-camera system are obtained by using the camera calibration toolbox for Matlab ${ }^{\circledR}[7]$.

\subsection{Marker Detection}

The detection of the color markers represents the extraction of distinct colors in given images from a CCD camera. Since each marker is distinguishable by their different features, the precise position of the markers can be extracted. This paper employs the RGB color-based marker detection algorithm. In the first place, the original image is decomposed into the RGB color space $(256,256,256)$. Then, each pixel of the image has three color channels whose value varies from 0 to 255 . The colors of the onboard markers used are red, green, blue and yellow. Since they depend largely on the lighting condition, shadow and noise, a threshold process is required to detect and classify them. The threshold condition of each color marker is determined by analyzing various viewpoints and illumination conditions. After a threshold process (Fig. 3.1 (b)), smoothing and morphology (Fig. 3.1 (c)) are followed to delete the noise blobs. By selecting the largest shape and computing the coordinates of center point of the marker, marker is detected as shown in Fig 3.1 (d). In addition, for reducing the image processing time, recursive target tracking method is introduced. Once the marker is detected, the search is performed within the ROI (region of interest, Fig. 3.1 (e)) which is small rectangular region around the marker center point. If the marker is not detected, the searching algorithm goes back to the initial step for searching full image. 


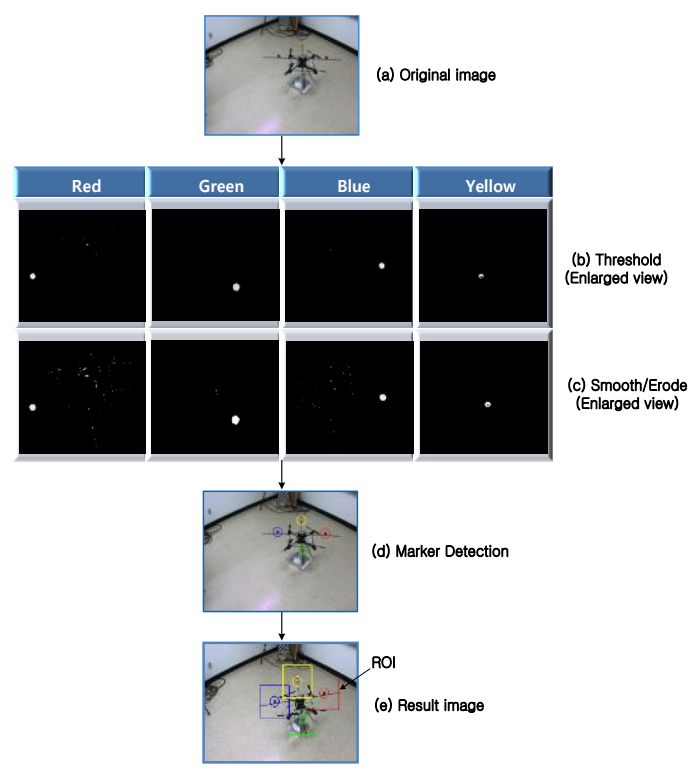

Figure 3.1 Color marker detection process

\subsection{Multi-UAV Tracking}

For tracking of multiple UAVs, it is difficult to use the same method as described in section 3.2 since the number of color which is able to extract is limited or when using the same color maker set for each UAV, which color marker is originated from which UAV should be decided. Figure 3.2 shows the example of color marker detection of two UAVs. Since the two same color makers are detected in each camera, the association of measurement (color marker) for UAV tracking is required. This problem is referred to as the data association and has extensively studied in the target tracking and surveillance community [8-10]. A number of data association techniques have been developed such as nearest neighbor, the track-splitting filter, joint-likelihood integer programming, multiple-hypothesis algorithm and the joint-probabilistic data association algorithm [11].

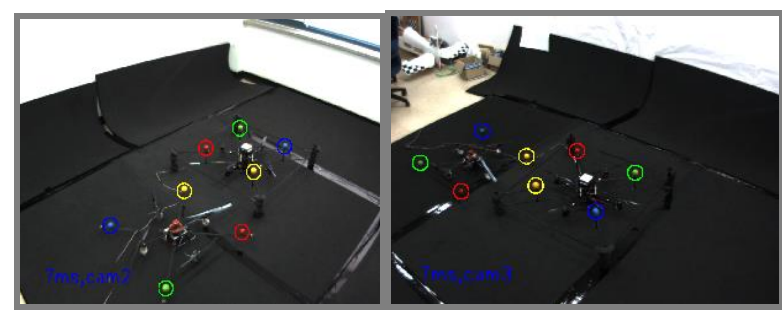
(a) Image of camera 1
(b) Image of camera 2 


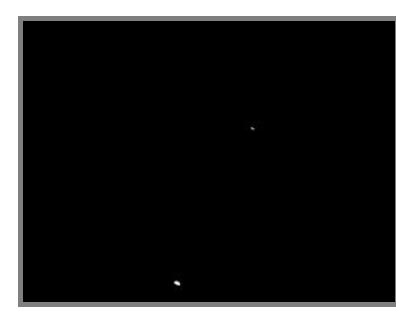

(c) Green markers in cam1

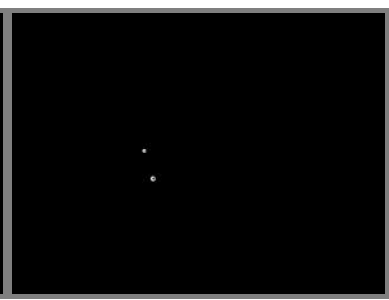

(d) Yellow markers in cam2

Figure 3.2 Marker detection of two UAVs

In this study, considering real-time operation environment, the nearest neighbor algorithm is used to associate the color marker to the related UAV at each camera independently (i.e. single camera tracking). When an ambiguity of the marker occurs at one camera, the epipolar geometry which uses the characteristic of the multi-camera system is employed to resolve the ambiguity [6].

\subsubsection{Nearest Neighbor Algorithm}

Nearest neighbor (NN) algorithm is the simplest data association algorithm based on the Kalman filter. The details of the NN algorithm are as follows. To begin with, the process model of the UAV is the second-order linear model with a constant speed and the measurement is the image coordinates of the marker detected by the camera. Assuming that the initial position of each marker is known and the process noise and measurement noise are normally distributed, each marker is tracked by the Kalman filter independently. Finally, the measurement which is the closest to its predicted value of the Kalman filter is selected, where the closest is defined by Mahalanobis distance (MD) [11]. MD can be considered as a generalization of the Euclidean distance which accounts for the relative uncertainties error estimate and generates the ellipsoidal validation volume related to the probability of finding measurement. Although the single camera tracking using $\mathrm{NN}$ algorithm is simple and easy to implement in real-time, it has finite chance that the association is incorrect. In case that color markers are very close as shown in Fig. 3.3 (b), the overlapping of the validation region occurs and results in wrong maker tracking. 


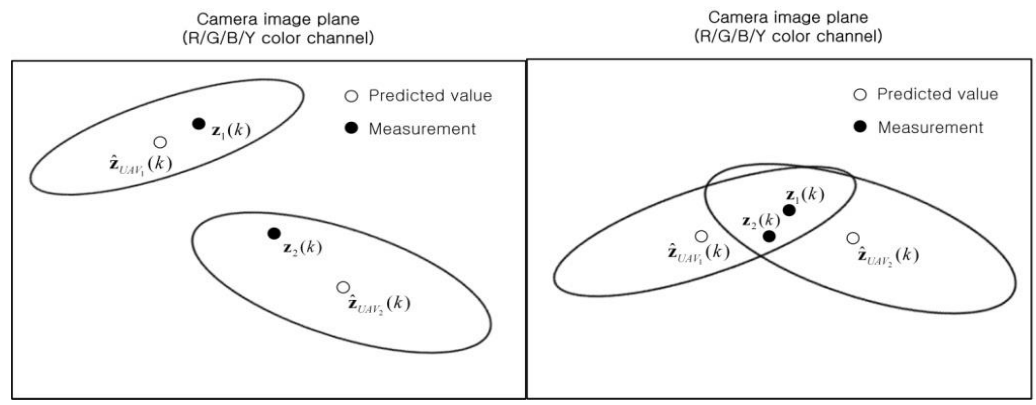

(a) No ambiguity

(b) Overlapping of validation volume

Figure 3.3 Ambiguity of single camera tracking using NN algorithm

\subsubsection{Epipolar Geometry}

To solve the ambiguity of the single camera tracking with $\mathrm{NN}$ algorithm, the epipolar geometry based on the constraint of the multiple view geometry is introduced additionally. The epipolar geometry is the geometry between two cameras, which consists of an epipole $\mathbf{e}^{\prime}$ (the point of intersection of the line joining the camera center), an epipolar plane $\mathbf{H}_{\pi}$ (a pane containing the base line), an epipolar line $\mathbf{l}^{\prime}$ (the intersection of an epipolar plane with the image plane) as shown in Fig. 3.4 [6].

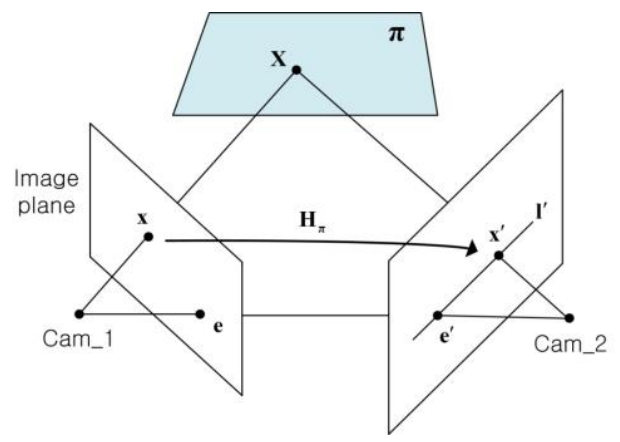

Figure 3.4 Epipolar geometry [6]

Figure 3.5 shows the example of the epipolar geometry. The two red markers of camera 2 are represented as the epipolar line at camera 1 and the blue and the green marker of camera 1 are represented as the epipolar line at camera 2 . It is shown that each marker lies on its corresponding epipolar line. 


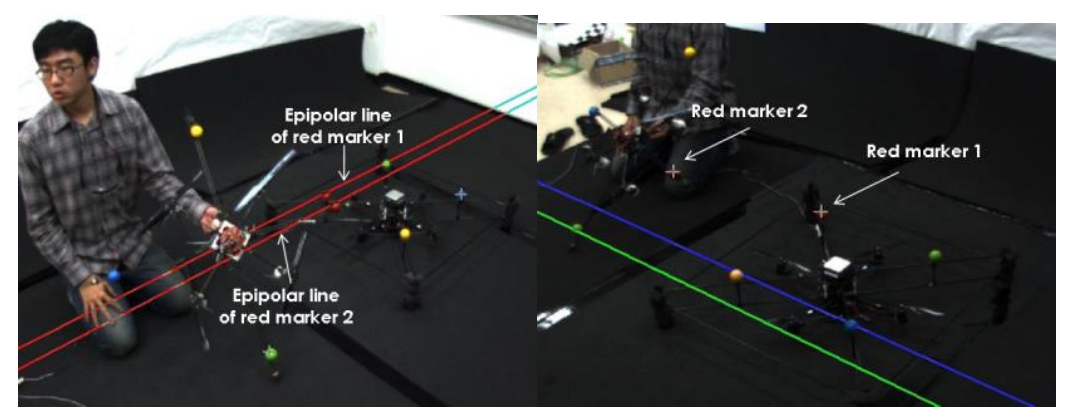

(a) Image of camera 1

(b) Image of camera 2

Figure 3.5 Example of the epipolar line

To use epipolar geometry for tracking, the correspondence condition is used and given as:

$$
\mathbf{x}^{\prime T} F \mathbf{x}=0
$$

where $F$ is the fundamental matrix which represents a mapping from a $2 \mathrm{D}$ onto 1D projective space. Since the point $\mathbf{x}^{\prime}$ corresponding to the point $\mathbf{x}$ lies on the epipolar line, the correspondence condition should be satisfied. In case that the ambiguity or occlusion of the color marker occurs at one camera, color marker can be distinguished by using the color marker information of the other camera obtained by $\mathrm{NN}$ algorithm and the correspondence condition. The overall algorithm of multi-UAV tracking is as follows. First, the RGB-based color marker detection is performed and the position of color marker is predicted by using Kalman filter with linear marker model. Then, the closest measurement to its predicted value is selected by computing $\mathrm{MD}$ (NN algorithm). When the ambiguity of the color marker occurs at one camera, data association is performed by using epipolar geometry. Figure 3.6 shows the flow chart of the multi-UAV tracking algorithm. 


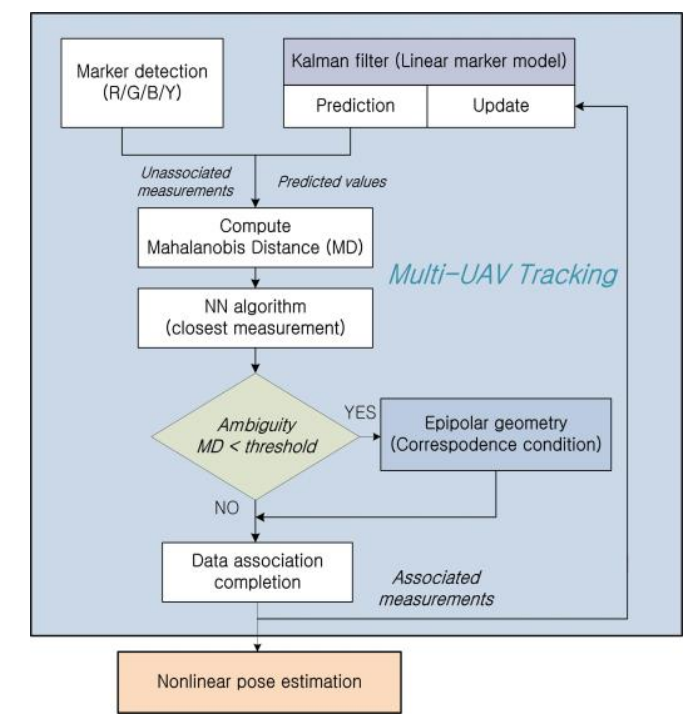

Figure 3.6 Flow chart of the multi-UAV tracking algorithm

\section{Pose Estimation}

For the pose estimation of the quad-rotor UAV, the following section gives details of the process model with twelve states and measurement model including its various noise sources. The estimation of the state is performed by the extended Kalman filter considering the delayed measurement.

\subsection{Dynamic Model}

The flat-Earth, body axes 6-DOF equations [12] used for conventional aircraft control design is adopted to describe the motion of the quad-rotor UAV. The state vector comprises the position $\left(X_{v}, Y_{v}, Z_{v}\right)$, velocity $(U, V, W)$, Euler angle $(\phi, \theta, \psi)$ and angular rate $(p, q, r)$ and defined as:

$$
\mathbf{X}=\left[\begin{array}{llllllllllll}
X_{v} & Y_{v} & Z_{v} & U & V & W & \phi & \theta & \psi & p & q & r
\end{array}\right]^{T}
$$

Since this study considers UAV flying near hover condition, it is assumed that the external force balances the gravity and there is no driving torques. This is reasonable for the indoor flight. The uncertainty from these assumptions is considered as zero-mean white Gaussian noise $w$ with the covariance matrix $Q$. Then, the dynamic model of the UAV for pose estimation can be written as: 


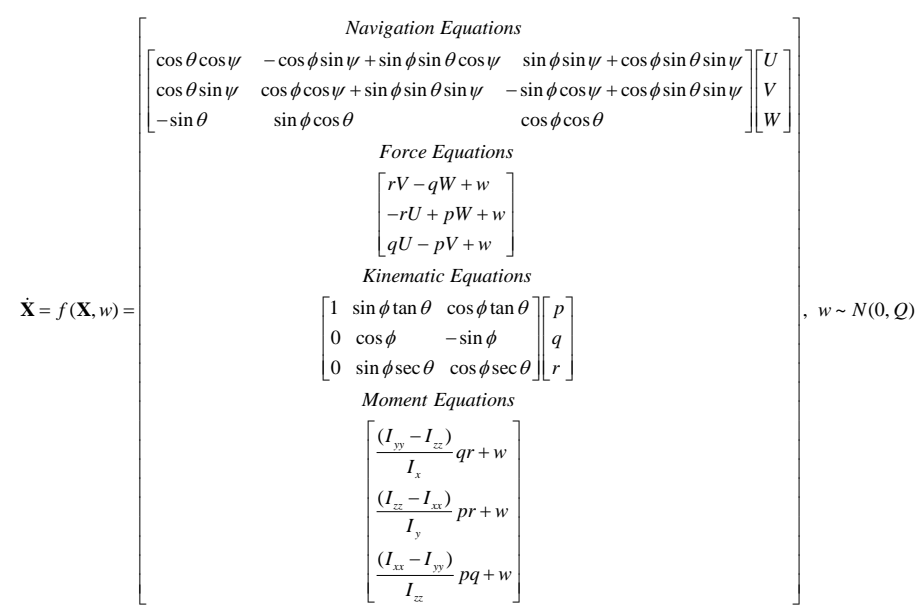

\subsection{Measurement Model}

The coordinates of the indoor test-bed system is represented in Fig. 4.1. The measurements are the $2 \mathrm{D}$ visual information in the image coordinates of each camera as:

$$
\begin{aligned}
& \mathbf{z}=\left[\begin{array}{llll}
z_{1} & z_{2} & z_{3} & z_{4}
\end{array}\right]^{T} \\
& \text { where, } z_{i}=\left[\begin{array}{llll}
x_{i}^{c a m 1} & y_{i}^{\text {cam } 1} & x_{i}^{\text {cam } 2} & y_{i}^{\text {cam } 2}
\end{array}\right]^{T}, i \in(1,2,3,4): \text { marker }
\end{aligned}
$$

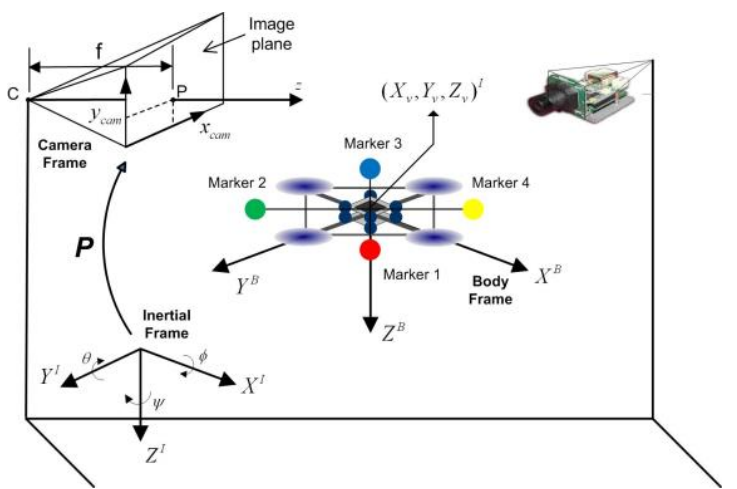

Figure 4.1 Geometry among UAV, camera and environment

The measurement model can be expressed as nonlinear equation using the rotation transform matrix and camera projection matrix. First of all, the positions of four markers with respect to the inertial frame are determined by using the position $\left(X_{v}, Y_{v}, Z_{v}\right)$ and Euler angles $(\phi, \theta, \psi)$ of the UAV and the pre-defined relative positions of the markers as: 


$$
\overline{\mathbf{X}}_{p t, i}^{I}=\left[X_{v} Y_{v} Z_{v}\right]^{T}+R_{B}^{I} \overline{\mathbf{X}}_{p t, i}^{B}, i \in(1,2,3,4): \text { marker }
$$

where $\overline{\mathbf{X}}_{p t, i}$ represents the 3D position of $i$-th marker, $I$ denotes an inertial frame, $B$ denotes a body frame and $R_{B}^{I}$ is rotation transform matrix from body to inertial frame. Then, the positions of markers are transformed into 2D visual information in the image coordinates of the camera by using the camera projective matrix as:

$$
\begin{aligned}
& \mathbf{z}=\left[\begin{array}{llll}
z_{1} & z_{2} & z_{3} & z_{4}
\end{array}\right]^{T} \\
& \text { where, } z_{i}=\left[\frac{P_{1}^{c a m, l}, \tilde{\mathbf{X}}_{p t, i}}{P_{3}^{c a m,}, 1 \tilde{\mathbf{X}}_{p t, i}} \frac{P_{2}^{c a m,}, \tilde{\mathbf{X}}_{p t, i}}{P_{3}^{c a m, l}, \tilde{\mathbf{X}}_{p t, i}} \frac{P_{1}^{c a m,}, \tilde{\mathbf{X}}_{p t, i}}{P_{3}^{c a m,}, \tilde{\mathbf{X}}_{p t, i}} \frac{P_{2}^{c a m,}, 2}{P_{3}^{c a m, 2}, \tilde{\mathbf{X}}_{p t, i}}\right]_{p t, i}^{T}, i \in(1,2,3,4): \text { marker }
\end{aligned}
$$

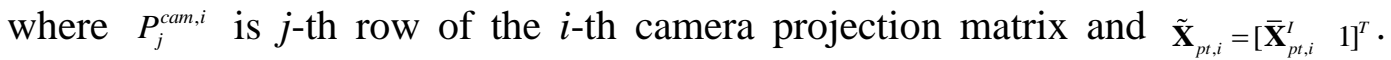
The final measurement equation is obtained by incorporating the measurement noise $v_{k}$ into Eq. (4.5) as:

$$
\mathbf{z}_{k}=h_{k}\left(\mathbf{X}_{k}\right)+v_{k}, v_{k} \sim N\left(0, R_{k}\right)
$$

Camera measurement noise is incurred by various sources such as calibration error, CCD sensor noise, marker detection error and time delay as shown Fig. 4.2, and can be modeled approximately as Gaussian distribution [13]. In this study, a zero mean Gaussian noise with the covariance matrix $R$ is used for the measurement noise.

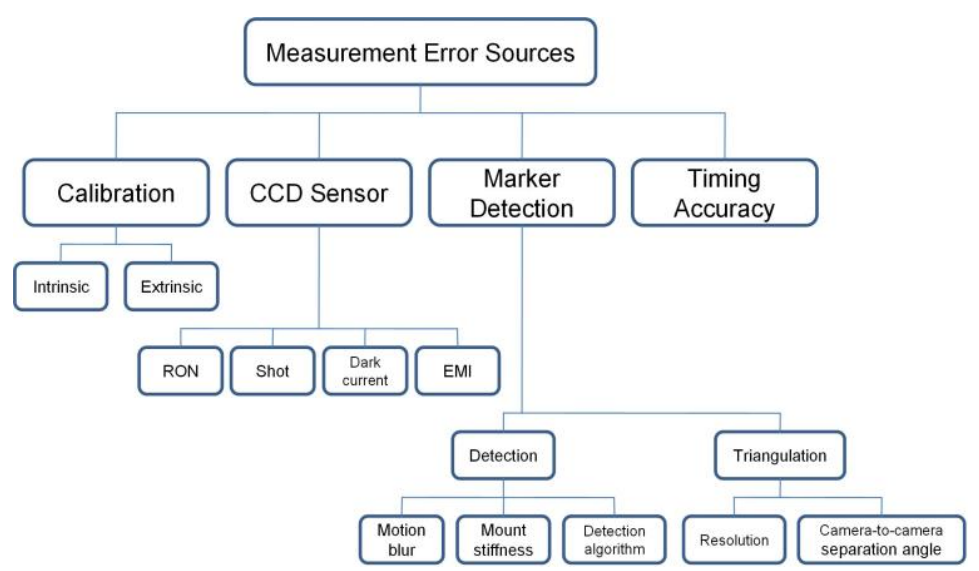

Figure 4.2 Measurement error sources 


\subsection{Nonlinear Estimation}

The extended Kalman filter (EKF) is used to estimate the state variables of the UAV. The EKF is a widely-used filtering method in tracking and control problems, which linearizes all nonlinear process and measurement models and applies the traditional Kalman filter. The EKF algorithm consists of the prediction and update stage as follows [14].

\section{$\underline{\text { Prediction }}$}

Integrate the state estimate and its covariance from time $(k-1)^{+}$to time $k^{-}$as follows:

$$
\begin{aligned}
& \dot{\mathbf{L}^{\prime}}=f(\mathbf{X}, 0) \\
& F(t)=\left.\frac{\partial f}{\partial \mathbf{X}}\right|_{\hat{\mathbf{X}}} \\
& \dot{\Phi}\left(t, t_{k-1}\right)=F(t) \Phi\left(t, t_{k-1}\right), \Phi\left(t_{k}, t_{k}\right)=I \\
& P_{k}^{-}=\Phi\left(t_{k}, t_{k-1}\right) P_{k-1}^{+} \Phi\left(t_{k}, t_{k-1}\right)^{T}+Q_{k-1}
\end{aligned}
$$

where $F$ and $\Phi$ denotes Jacobian matrix of the process model and state transition matrix, respectively. This integration process is started with the relation,

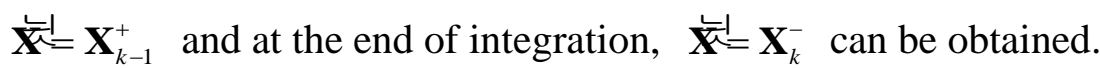

\section{$\underline{\text { Update }}$}

At time $\mathrm{k}$, incorporate the measurement $y_{k}$ into the state estimate and covariance estimate as:

$$
\begin{aligned}
H_{k} & =\left.\frac{\partial h_{k}}{\partial \mathbf{X}}\right|_{\hat{\mathbf{X}}_{k}^{-}} \\
P_{k}^{+} & =\left[\left(P_{k}^{-}\right)^{-1}+H_{k}^{T} R_{k}^{-1} H_{k}\right]^{-1} \\
K_{k} & =P_{k}^{+} H_{k}^{T} R_{k}^{-1} \\
\text { 育 } & =\mathbf{X}_{k}^{-}+K_{k}\left[\mathbf{z}_{k}-h_{k}\left(\mathbf{X}_{k}^{-}\right)\right]
\end{aligned}
$$

where $H_{k}$ and $K_{k}$ denote Jacobian matrix of the measurement model and a Kalman gain, respectively. The EKF provides the reasonably accurate navigation information by optimally tuning the information between the uncertain vehicle dynamics and the camera measurement. However, since there is a time difference 
between an actual marker motion and image data due to the image process and data communication, the EKF considering measurement delay is considered additionally. Although there are various ways to deal with delayed measurements [15-17], this study uses the method proposed in [17] under the assumption of constant time delay. The measurement is assumed to be transmitted from the camera at time s and arrived with a delay $(\Delta)$ at time $k=s+\Delta$ as shown in Fig. 4.3.

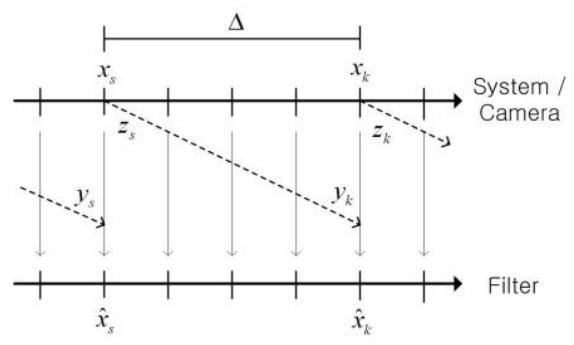

Figure 4.3 Delayed measurements

The measurement equation at time $k$ becomes:

$$
\mathbf{y}_{k}=\mathbf{y}_{s+\Delta}=\mathbf{z}_{s}=h_{s}\left(\mathbf{X}_{s}\right)+v_{s}
$$

where $\mathbf{z}$ is the measurement from camera and $\mathbf{y}$ is the measurement into the filter. Then, the update stage of the extended Kalman filter is modified as:

$$
\begin{aligned}
H_{k}= & \left.\frac{\partial h_{s}}{\partial \mathbf{X}}\right|_{\hat{\mathbf{x}}_{s}^{-}} \\
K_{k}= & {\left[P_{k}^{-}-Q(k, s)\right]\left(H_{s} \Phi^{-1}(k, s)\right)^{T}\left\{H_{s} \Phi^{-1}(k, s)\right.} \\
& {\left.\left[P_{k}^{-}-Q(k, s)\right] H_{s} \Phi^{-1}(k, s)^{T}+R(k, s)\right\}^{-1} } \\
P_{k}^{+}= & {\left[I-K_{k} H_{s} \Phi^{-1}(k, s)\right]\left[P_{k}^{-}-Q(k, s)\right]+Q(k, s) } \\
\text { 厸 } & =\mathbf{X}_{k}^{-}+K_{k}\left[\mathbf{z}_{s}-h_{s}\left(\Phi^{-1}(k, s) \mathbf{X}_{k}^{-}\right)\right]
\end{aligned}
$$

\section{Quad-rotor UAV Controller Design}

\subsection{Quad-rotor UAV Modeling}

The quad-rotor UAV consists of a rigid cross frame equipped with four rotors. The quad-rotor generates its motion by only controlling the angular velocity of 
each rotor. For the quad-rotor UAV control, the 6-DOF equations used in Eq. (4.2) is used with the external forces $\left(F_{x}, F_{y}, F_{z}\right)$ and the external moments $(L, M$, $N$ ) acting on the center of gravity with respect to body-fixed frame as:

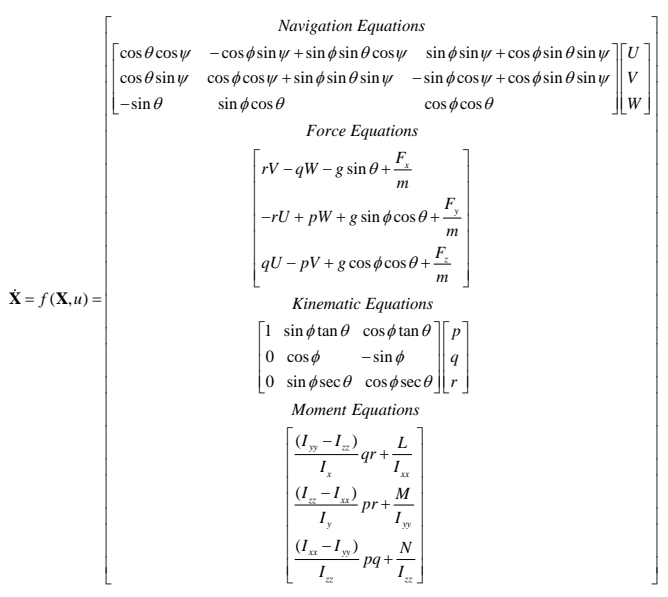

In the quad-rotor UAV, the external forces and moments are generated by the aerodynamic forces of four rotors. Under the assumption that the aerodynamic coefficients of rotors are constant, the external forces and moments are obtained as follows [18]. First, the thrust generated by the rotor $\mathrm{i}$ is written as:

$$
T=K_{t} \Omega_{i}^{2}
$$

where $K_{t}$ is thrust coefficient and $\Omega$ denotes the angular velocity of the rotor. Since the thrust acts on z-axis only, the external forces are given by:

$$
\begin{aligned}
& F_{x}=0 \\
& F_{y}=0 \\
& F_{z}=-K_{t}\left(\Omega_{1}{ }^{2}+\Omega_{2}{ }^{2}+\Omega_{3}{ }^{2}+\Omega_{4}{ }^{2}\right)
\end{aligned}
$$

The reactive torque generated by the rotor i due to rotor drag is given by:

$$
\tau_{r}=K_{r} \Omega_{i}^{2}
$$

where $K_{r}$ is torque coefficient. Then, the airframe torque generated by the rotors is given by: 


$$
\boldsymbol{\tau}_{a}=\left[\begin{array}{c}
K_{t}\left(-\Omega_{2}^{2}+\Omega_{4}^{2}\right) d \\
K_{t}\left(-\Omega_{1}^{2}+\Omega_{3}^{2}\right) d \\
K_{r}\left(-\Omega_{1}{ }^{2}+\Omega_{2}{ }^{2}-\Omega_{3}{ }^{2}+\Omega_{4}{ }^{2}\right)
\end{array}\right]
$$

where $d$ is the distance from the rotors to the center of mass of the quad-rotor. The gyroscopic toques due to the combination of the rotation of the airframe and the four rotors are given by:

$$
\boldsymbol{\tau}_{g}=\sum_{i=1}^{4}\left(\mathbf{w} \times \mathbf{e}_{\mathbf{z}}\right)(-1)^{i+1} I_{r} \Omega_{i}
$$

where $\mathbf{e}_{z}=[0,0,1]^{T}$ denotes the unit vector, $\mathbf{w}$ is the angular velocity vector of the airframe expressed in the body frame, and $\mathbf{w}=[p, q, r]^{T}$ to be specific. $I_{r}$ is the inertia of the rotor. Adding airframe and gyroscopic torque, the external moments are obtained and given by:

$$
\begin{aligned}
& L=K_{t}\left(-\Omega_{2}^{2}+\Omega_{4}^{2}\right) d+I_{r} q\left(\Omega_{1}-\Omega_{2}+\Omega_{3}-\Omega_{4}\right) \\
& M=K_{t}\left(-\Omega_{1}^{2}+\Omega_{3}^{2}\right) d-I_{r} p\left(\Omega_{1}-\Omega_{2}+\Omega_{3}-\Omega_{4}\right) \\
& N=K_{r}\left(-\Omega_{1}{ }^{2}+\Omega_{2}{ }^{2}-\Omega_{3}{ }^{2}+\Omega_{4}{ }^{2}\right)
\end{aligned}
$$

An actuator in quad-rotor control system is the DC motor. The dynamics of a DC motor system is assumed as a first order system [19] and its transfer function is given by:

$$
G(s)=\frac{1}{\tau s+1}
$$

where $\tau$ is the time constant of motor dynamics.

\subsection{Classical Control System Design}

The entire control architecture for the quad-rotor UAV is as shown in Fig. 5.1. In inner loop, Euler angles and angular velocities are looped back to the position hold autopilot. In outer loop, position and velocities are looped back to the position hold autopilot. 


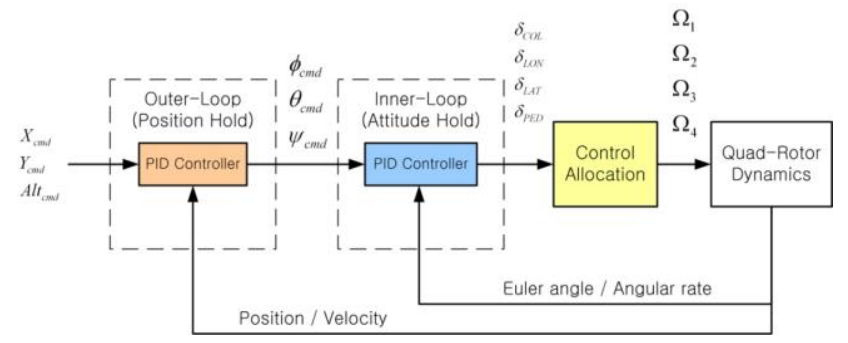

Figure 5.1 Control architecture

Four control channel commands generated by the controller are transformed into the angular velocity of each rotor by using control allocation method as given:

$$
\begin{aligned}
& \Omega_{1}=\Omega_{n o m}+\left(\delta_{C O L} / 4\right)+\left(\delta_{L O N} / 2\right)-\left(\delta_{P E D} / 4\right) \\
& \Omega_{2}=\Omega_{n o m}+\left(\delta_{C O L} / 4\right)-\left(\delta_{L A T} / 2\right)+\left(\delta_{P E D} / 4\right) \\
& \Omega_{3}=\Omega_{n o m}+\left(\delta_{C O L} / 4\right)-\left(\delta_{L O N} / 2\right)-\left(\delta_{P E D} / 4\right) \\
& \Omega_{4}=\Omega_{\text {nom }}+\left(\delta_{C O L} / 4\right)+\left(\delta_{L A T} / 2\right)+\left(\delta_{P E D} / 4\right)
\end{aligned}
$$

where $\Omega_{\text {nom }}$ is the nominal angular velocity of rotor and $\delta_{C O L}, \delta_{L O N}, \delta_{L A T}$ and $\delta_{P E D}$ is collective, longitudinal, lateral and directional control input, respectively. The attitude hold autopilot is designed to track and hold the pitch, roll and yaw angle. It consists of the inner-loop with an angular rate feedback and the outer loop with the Euler angles feedback by PD control concept. The block diagram of the attitude hold autopilot is shown in Fig. 5.2

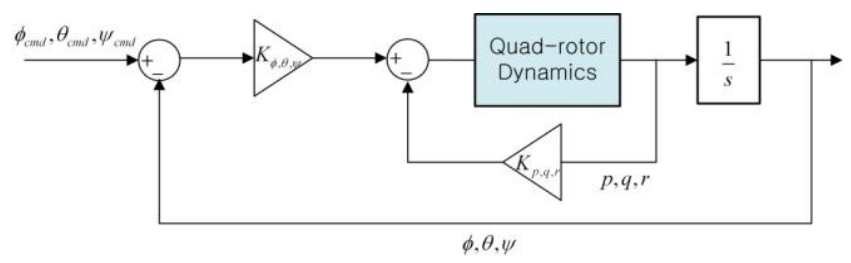

Figure 5.2 Block diagram of the attitude hold autopilot

Position hold is achieved by the pitch and roll attitude control, respectively. Control law for the position hold autopilot is given by:

$$
\begin{gathered}
\theta_{c m d}=K_{X}\left(X_{c m d}-X\right)-K_{v_{X}} V_{X} \\
\phi_{c m d}=K_{Y}\left(Y_{c m d}-Y\right)-K_{v_{Y}} V_{Y}
\end{gathered}
$$


Altitude hold is achieved by collective control input directly.

$$
\left(\delta_{C O L}\right)_{c m d}=K_{h}\left(h_{c m d}-h\right)-K_{v_{h}} V_{h}
$$

\section{Experiment Results}

This section presents the performance of the vision and pose estimation algorithm. Experiments are carried out using the Multi-Agent Test-bed for Real-time Indoor eXperiment (MATRIX) system.

\subsection{Test-Bed Configuration}

Indoor flight test-bed called MATRIX is developed as shown in table 6.1 and Fig. 6.1. Two firewire CCD cameras with a horizontal FOV of $56.1^{\circ}$ and external triggering board provide the synchronized images of the UAV from different field of views to the ground computer. The ground control system is designed to check the image data, processing time, marker detection, rotor speed, attitude heading reference system (AHRS) data and pose estimation results.

Table 6.1 MARIX specification

\begin{tabular}{c|c|c}
\hline \hline \multicolumn{2}{c}{ MARIX Specification } \\
\hline \hline \multirow{4}{*}{$\begin{array}{c}\text { Multi- } \\
\text { Camera } \\
\text { System }\end{array}$} & Number & 2 Firewire CCD Cameras \\
\cline { 2 - 3 } & Field of View (FOV) & 1024 X 768 \\
\cline { 2 - 3 } & Frames per Second (FPS) & $36.1^{\circ}$ (horizontal) / 43.6 (vertical) \\
\cline { 2 - 3 } & Height & $1.40 \mathrm{~m}$ \\
\cline { 2 - 3 } & Distance & $2.20 \mathrm{~m}$ \\
\hline & Triggering Board & NI DAQ PCI-6602 \\
\hline Ground Computer & Quad-rotor UAV \\
\hline
\end{tabular}




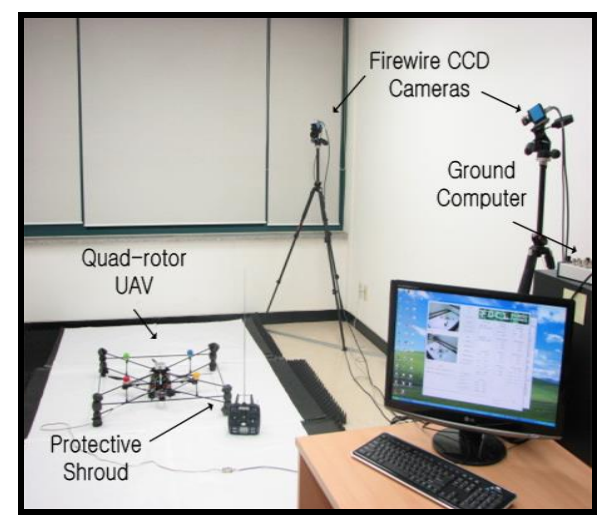

Figure 6.1 MATRIX system

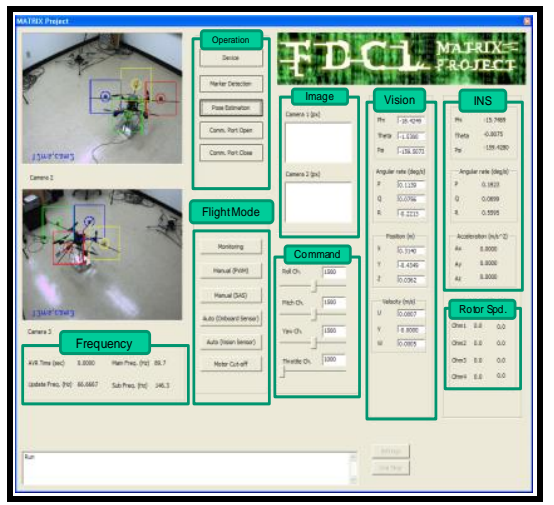

Figure 6.2 Ground control system

The quad-rotor UAV used in experiment weighs $1.1 \mathrm{~kg}$ with the width of $0.72 \mathrm{~m}$ and height of $0.15 \mathrm{~m}$. To enhance the durability and the safety of the quad-rotor $\mathrm{UAV}$, the protective shroud is made as shown in Fig. 6.1. The protective shroud is the frame of light weight and high strength carbon fiber tubes joined by plastic joints. The quad-rotor UAV consists main and sub micro controller unit (MCU) to control the angular velocity of rotor, inertial measurement unit (IMU), RF receiver, electric speed controller (ESC) and brushless DC motor (BLDC) as shown in Fig. 6.3. It communicates with the ground computer through either radio frequency (remote control command) or RS-232 cable (ground control system command) as shown in Fig. 6.4.

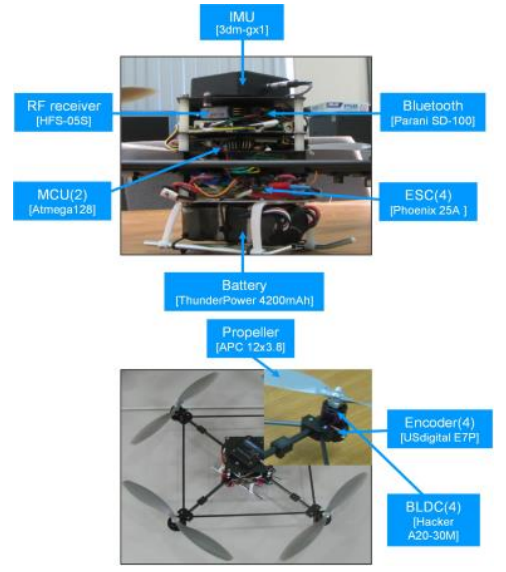

Figure 6.3 Quad-rotor UAV configuration

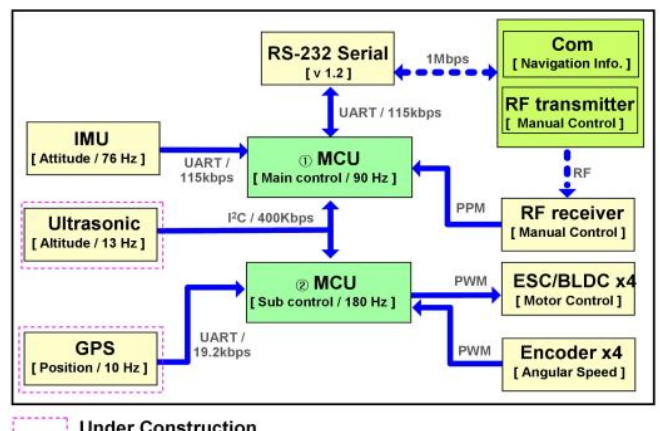

Figure 6.4 Quad-rotor UAV communication

The 3-DOF flying mill in Fig. 6.5 is designed to implement and tune the attitude controller. This flying mill gives the vehicle unrestricted yaw motion and about 45 degrees of pitch and roll motions, while restricting the vehicle to a fixed position in the three-dimensional space. Some of the factors are considered in developing 
the 3-DOF flying mill to improve the validity of experimental results. First, the ground effect occurred by four rotors in low altitude is solved by placing the 3DOF flying mill 0.7 meters above the ground. Second, additional four mass balancers are built to match the centers of spherical joint and the vehicle. Positions of these balancers are determined from estimating the moment of inertia by CATIA program. Finally, low friction spherical joint is employed to reduce its influence on the stability of rotational dynamics. These considerations make it possible to use of 3-DOF flying mill with acceptable level of validity.

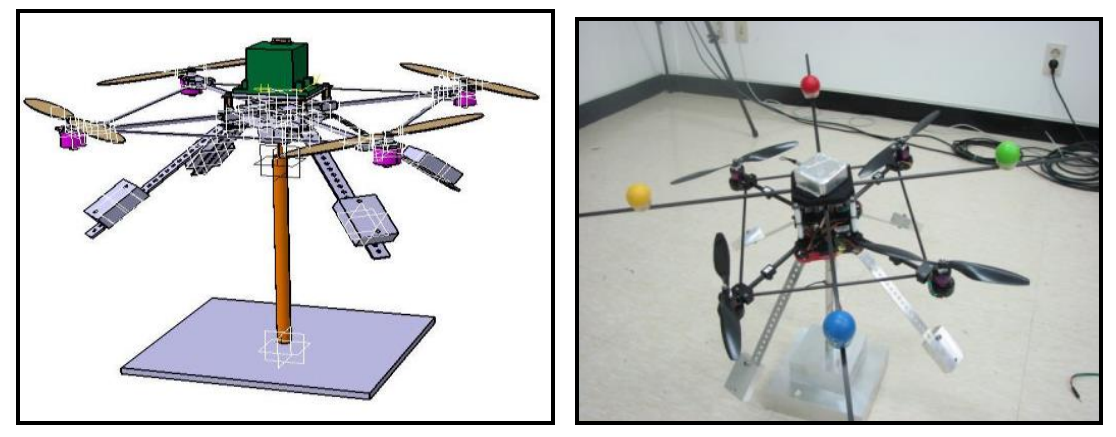

Figure 6.5 Quad-rotor UAV on the 3-DOF flying mill

\subsection{Multi-UAV Tracking Results}

Multi-UAV tracking experiment is performed on the condition that one UAV is moving manually while the other UAV is fixed and red markers of two UAVs are very close or occluded by each other as shown in Fig. 3.6. Figure 6.6 shows the results of marker tracking using only nearest neighbor algorithm which selects the measurement having the closest Mahalanobis distance (MD). After two red markers are occluded about 1.9 second at camera 1, even though there are two markers, data association algorithm selects one measurement having the closest MD for both predicted values of the red marker. On the contrary, Fig. 6.7 shows the results of marker tracking using $\mathrm{NN}$ algorithm and the correspondence condition of the epipolar geometry. The threshold parameter of MD is set to 9.21 which has $99 \%$ probability of finding measurement. In case that the MD of both red marker measurements is less than $9.21(1.5,4,8,11 \mathrm{sec})$ at camera 1 , which means that the overlapping of the validation volume occurs, the data association is accomplished successfully by additionally using the correspondence condition of the epipolar geometry as shown in Fig. 6.7 (a) and (b). Figure 6.7 (c) represents 
the position of the red marker of each UAV using proposed data association algorithm and linear triangulation method [6] which determines the 3D position with stereo image coordinates.
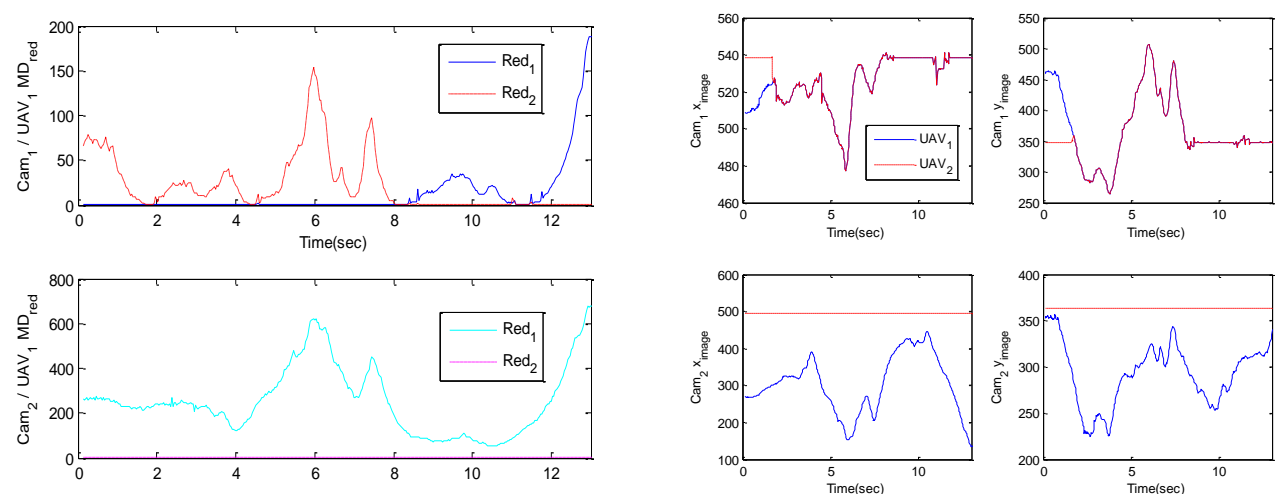

(a) MD of the red marker at each camera

(b) Image coordinates of the red color marker

Figure 6.6 Results of the NN algorithm
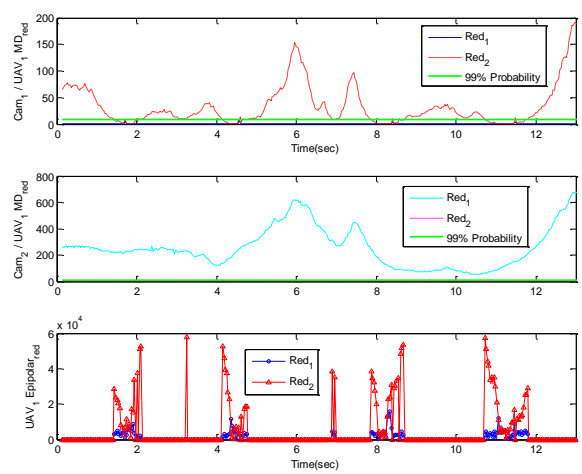

(a) MD of the red marker at each camera
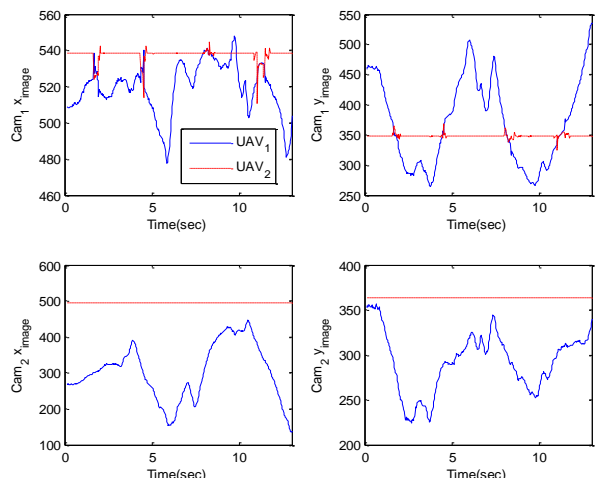

(b) Image coordinates of the red color marker

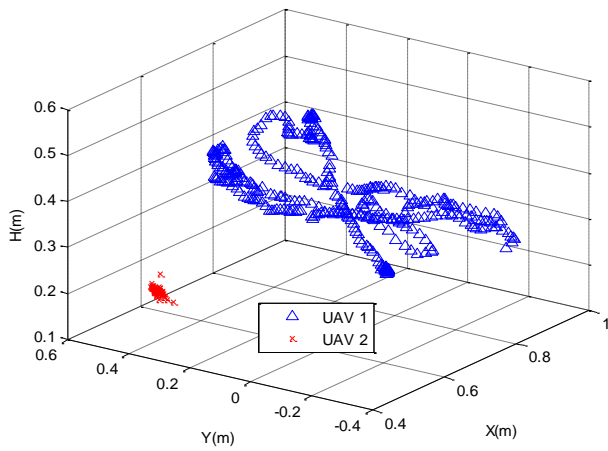

(c) 3D position of the red marker of each UAV

Figure 6.7 Results of NN algorithm and the epipolar geometry 


\subsection{Pose Estimation Results}

Before pose estimation, it is required to check the noise characteristic of the multicamera system to get an accurate design parameter of the filter. The noise of the measurement model can be obtained by analyzing the images of the markers since it depends on the performance of the CCD sensor and marker detection capability. Fig. 6.8 shows the histogram of the image coordinate of the red and yellow color marker of quad-rotor UAV at fixed position. Besides, the position of the UAV obtained by linear triangulation method and its histogram are shown in Fig. 6.9. This figure shows that the multi-camera system and vision algorithm has a high precision.
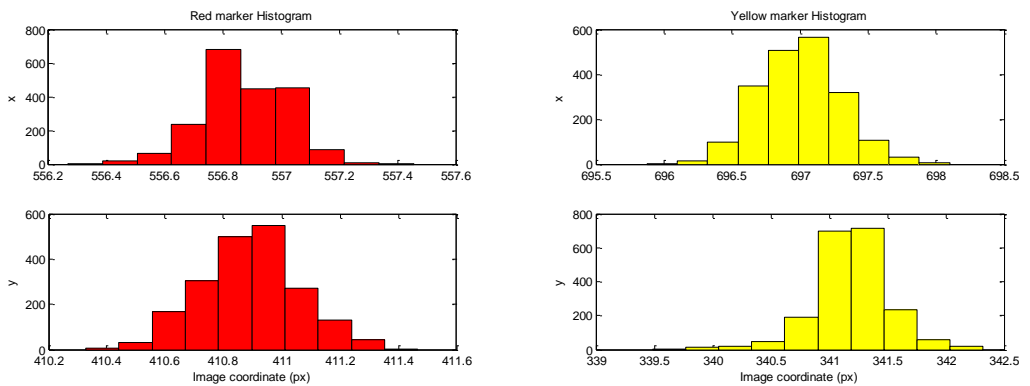

Figure 6.8 Histogram of the camera image coordinates of each marker at fixed position
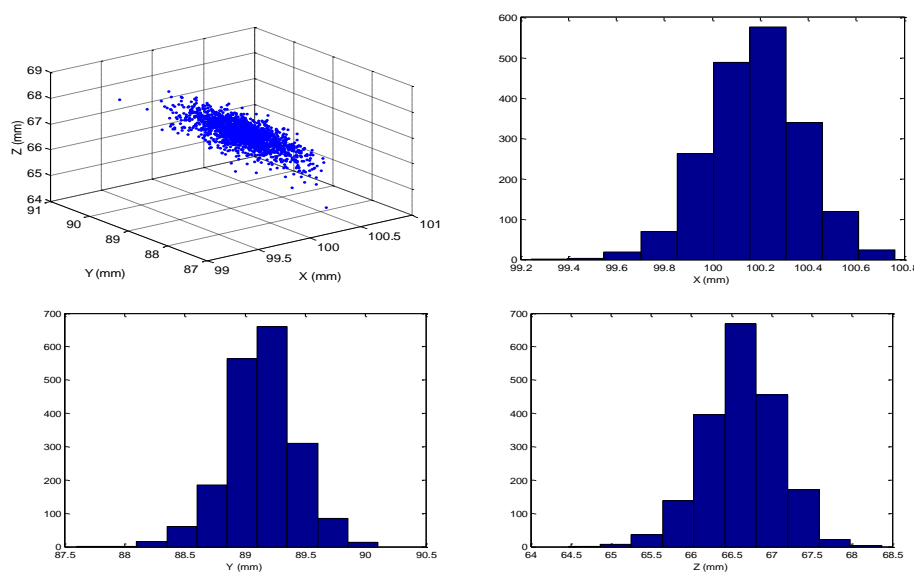

Figure 6.9 Histogram of UAV position at fixed position by triangulation

Pose estimation experiment is performed in case that the UAV is flying on the 3DOF flying mill. Measurement update rate is $30 \mathrm{~Hz}$ and process noise is the zeromean white Gaussian noise with the covariance of 0.001 . Figure 6.10 shows that the state estimation of the UAV using the EKF and position obtained by linear 
triangulation method and 3DM-GX1 AHRS measurement data at $\pm 2.0^{\circ}$ accuracy.
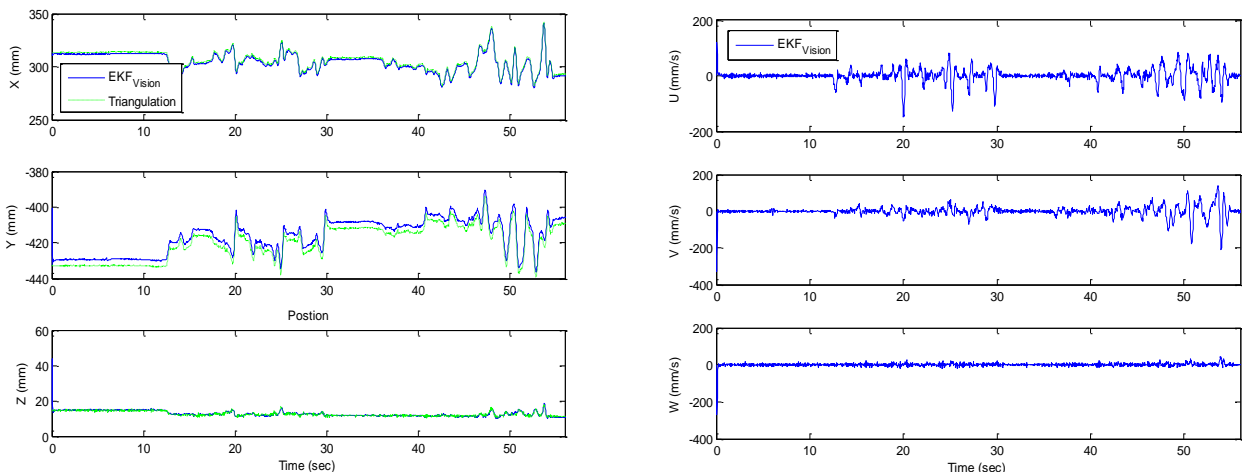

(a) Position (Vision/Triangulation)

(b) Velocity (Vision)
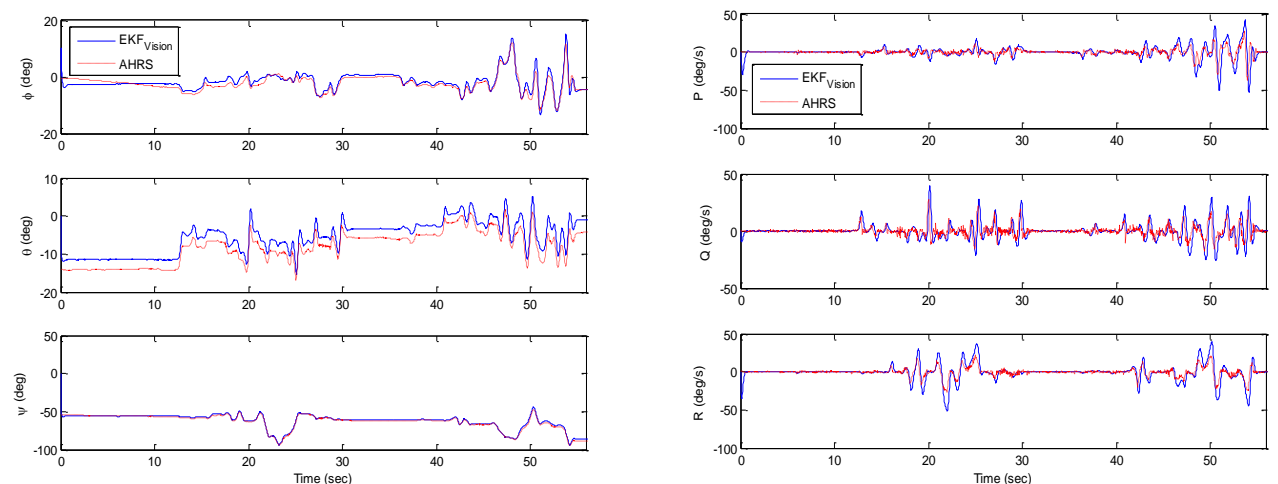

(c) Euler angles (Vision/AHRS)

(d) Body-axed angular rates (Vision/AHRS)

Figure 6.10 Pose estimation results

Table 6.2 shows the average and standard deviation for the bias error between estimated Euler angles and AHRS data. This is generated from calibration error of camera and can be decreased by the precise calibration procedure.

Table 6.2 Bias error

\begin{tabular}{ccc}
\hline \hline \multirow{2}{*}{ Attitude } & \multicolumn{2}{c}{$\mid$ Vision - AHRS | (deg) } \\
& Average & Standard deviation \\
\hline Roll & 1.039 & 0.611 \\
Pitch & 2.603 & 0.508 \\
Yaw & 0.9512 & 0.625 \\
\hline
\end{tabular}


Moreover, although there is the time delay caused by the image processing as shown in table 6.3, the effect of delay can be reduced by using modified EKF considering the delayed measurement as explained in section 4.3. Figure 6.11 shows Euler angles and angular rates comparison between EKF and modified EKF considering the delayed measurement. From the above results, it is verified that the MATRIX system has a reasonable performance, which can be used to control the quad-rotor UAV.

Table 6.3 Processing time

\begin{tabular}{cc}
\hline \hline Process & Processing time \\
\hline Image processing & $15 \sim 20 \mathrm{~ms}$ \\
Estimation algorithm & $5 \mathrm{~ms}$ \\
Communication & $5 \sim 10 \mathrm{~ms}$ \\
Miscellaneous & $\sim 5 \mathrm{~ms}$ \\
\hline Total & $\sim 40 \mathrm{~ms}$ \\
\hline \hline
\end{tabular}
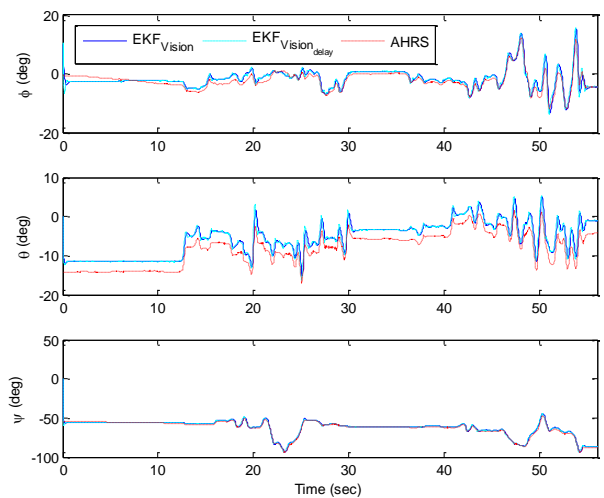

(a) Euler angles
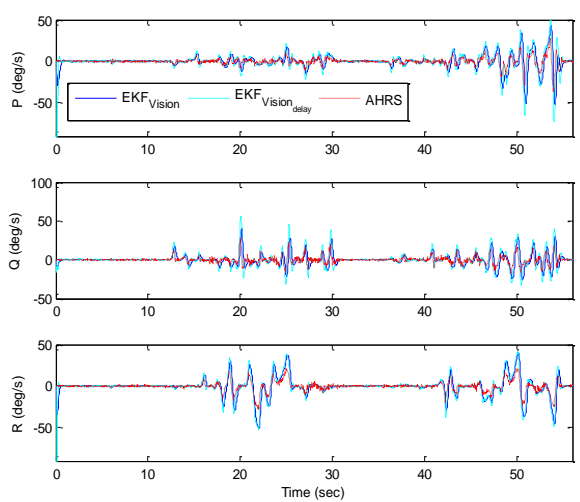

(c) Angular rates
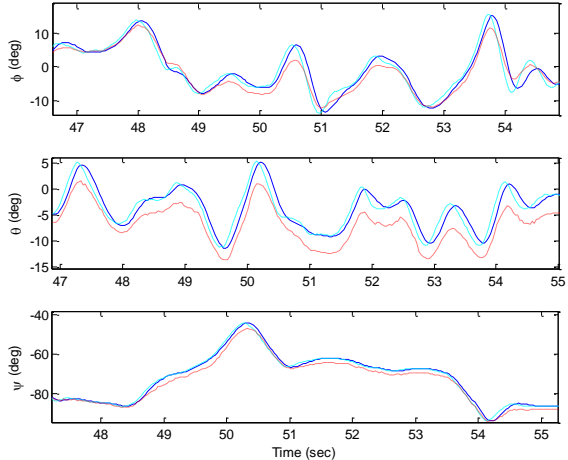

(b) Euler angles - enlarged view
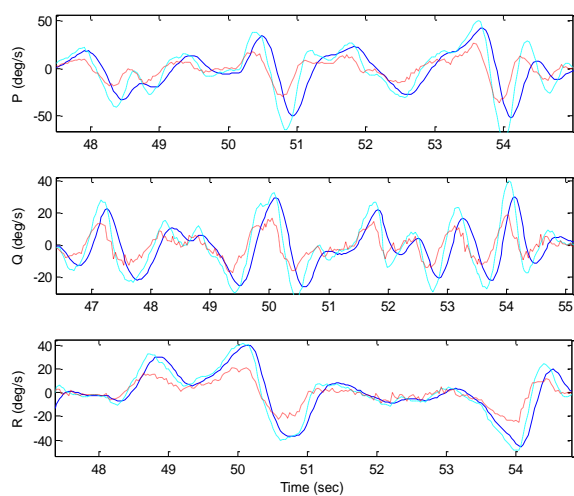

(d) Angular rates - enlarged view

Figure 6.11 Euler angles and angular rates estimation comparison between EKF and EKF considering delayed measurements 


\subsection{Flight Test Results}

\subsubsection{Attitude Stabilization}

For the attitude control, this study uses Euler angles estimated by EKF considering the delayed measurement in MATRIX system and angular rates from AHRS. Controller is designed by PD control concept as explained in section 5.3. Fig. 6.12 shows the experiment result of the attitude stabilization $\left(\phi=\theta=\psi=0^{\circ}\right)$ on 3-DOF flying mill. The performance represents that the proposed algorithm is enough to control the attitude of the indoor UAV. Control gains are as given in Table 6.4.
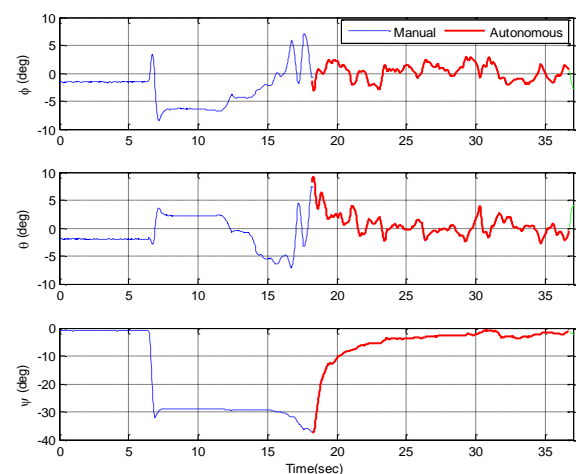

(a) Euler angles
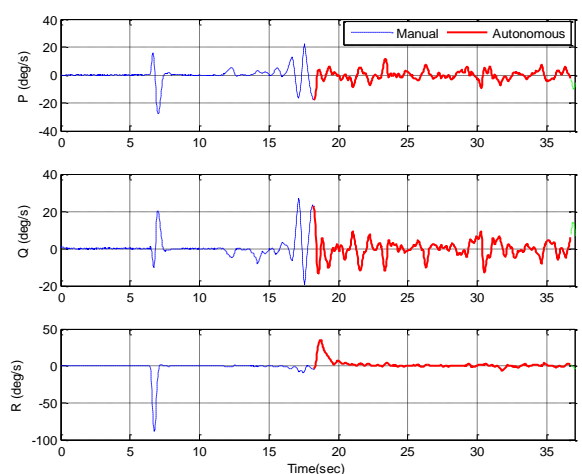

(b) Angular rates

Figure 6.12 Experiment results of the attitude stabilization on 3-DOF flying mill

(Euler angles from MATRIX and angular rates from AHRS)

Table 6.4 Control gains for the attitude hold autopilot

\begin{tabular}{cccccc}
\hline \hline \multicolumn{2}{c}{ Roll } & \multicolumn{2}{c}{ Pitch } & \multicolumn{2}{c}{ Yaw } \\
\hline$K_{p}$ & 160 & $K_{q}$ & 160 & $K_{r}$ & 850 \\
$K_{\phi}$ & 240 & $K_{\theta}$ & 240 & $K_{\psi}$ & 1150 \\
\hline \hline
\end{tabular}

\subsubsection{Position Control}

The position control is performed by using the position, velocity and attitude from MATRIX system and angular rates from AHRS. Control gains are shown in Table 6.5. Due to the effect on 3-DOF flying mill such as friction of ball bearing and inertia of balance mass bar, control gains of roll, pitch and yaw channels are a 
little different from those of Table 6.4 , but it is the same order of magnitude. Accordingly, it can be conclude that the experiment on the 3-DOF flying mill describes the real flight test properly within acceptable error bound.

Table 6.5 Control gains for the position hold autopilot

\begin{tabular}{cccccccccc}
\hline \hline \multicolumn{2}{c}{ Roll } & \multicolumn{2}{c}{ Pitch } & \multicolumn{2}{c}{ Yaw } & \multicolumn{2}{c}{ X } & \multicolumn{2}{c}{ Y } \\
\hline$K_{p}$ & 120 & $K_{q}$ & 120 & $K_{r}$ & 500 & $K_{V_{X}}$ & -0.18 & $K_{V_{Y}}$ & 0.18 \\
$K_{\phi}$ & 220 & $K_{\theta}$ & 220 & $K_{\psi}$ & 1250 & $K_{X}$ & -0.12 & $K_{Y}$ & 0.12 \\
\hline \hline
\end{tabular}

Fig. 6.13 shows that the flight test result of the position and the heading control without an altitude control (i.e. fixed throttle). The mean and standard deviations for tracking error between command and state are $0.056 \mathrm{~m}$ and $0.106 \mathrm{~m}$ for $\mathrm{x}$, $0.115 \mathrm{~m}$ and $0.215 \mathrm{~m}$ for $\mathrm{y}$ and $-0.577^{\circ}$ and $2.210^{\circ}$ for heading angle, respectively.

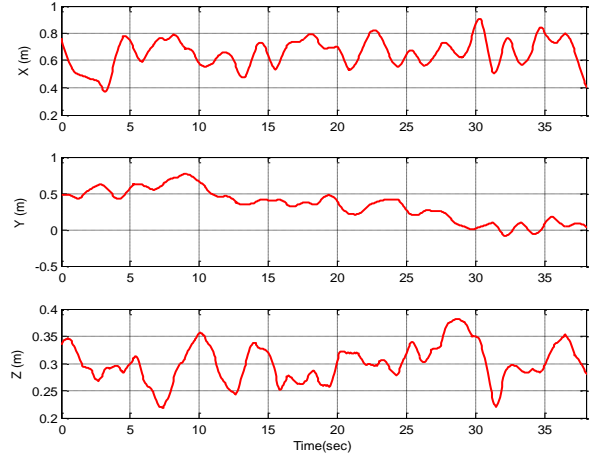

(a) Position $\left(X_{c m d}=0.6 \mathrm{~m} / Y_{\text {cmd }}=0.45 \mathrm{~m}\right)$
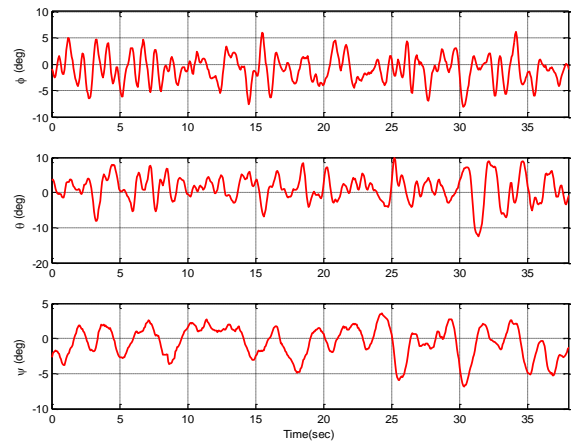

(c) Euler angles $\left(\psi_{c m d}=0^{\circ}\right)$
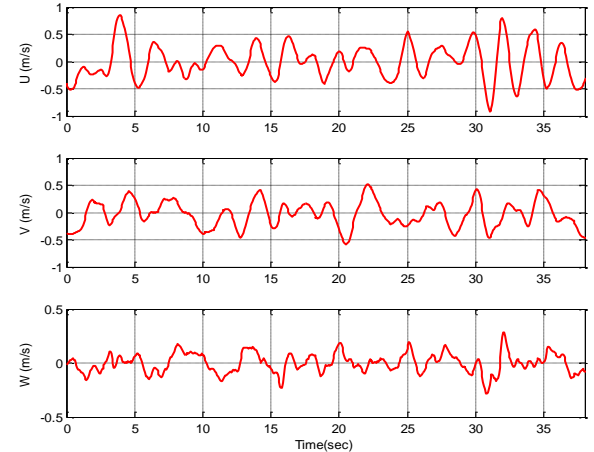

(b) Velocity
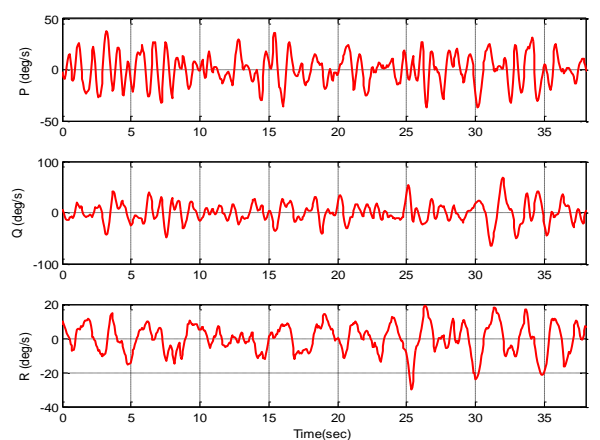

(d) Angular rates

Figure 6.13 Flight test results of the position control (Position/velocity/attitude from MATRIX and angular rates from AHRS) 
From the experiment result of pose estimation, attitude stabilization and position control, it is verified that the proposed MATRIX system can be applied to the autonomous flight control of the quad-rotor UAV.

\section{Conclusion}

In this paper, the control of the quad-rotor UAV using multi-camera visual feedback is presented. In the first place, the indoor flight test-bed that consists of multi-camera system, ground computer and the quad-rotor UAV is developed. In addition, the vision algorithm including camera calibration technique, color-based marker detection and nonlinear pose estimation method using the extended Kalman filter is introduced. The experiment results show that the proposed algorithm with the two-camera system provides an accurate and reliable pose estimation which can be used to control the quadrotor UAV. The significant contribution of this paper is the development of the indoor flight test-bed using only low-cost cameras allowing the full 6 DOF pose estimation and its application to the control of the quad-rotor UAV. The developed system, moreover, can be applied to validation of guidance and control algorithm for the multiple UAVs and various indoor autonomous missions such as reconnaissance and surveillance.

\section{Acknowledgement}

Authors are gratefully acknowledging the financial support by Agency for Defense Development and by UTRC (Unmanned Technology Research Center) and Brain Korea 21 Project, Korea Advanced Institute of Science and Technology.

\section{References}

1. M.Valenti, B.Bethke, D.Dale, A.Frank, J.McGrew, S.Ahrens, J.How, and J.Vian, "The MIT Indoor Multi-Vehicle Flight Testbed," 2007 IEEE International Conference on Robotics and Automation, pp.2758-2759, 2007.

2. E. Altug, J. P. Ostrowski, and C. J. Taylor, "Control of a Quadrotor Helicopter Using Dual Camera Visual Feedback," The International Journal of Robotics Research, Vol. 24, No. 5, pp. 329-341, May. 2005. 
3. Y. Yoshihata, K. Watanabe, Y. Iwatani, and K. Hashimoto, "Multi-camera visual servoing of a micro helicopter under occlusions," Proceedings on the IEEE/RSJ International Conference on Intelligent Robots and Systems, pp.2651-2620, 2007.

4. L.C. Mak, M. Whitty and T. Furukawa, "A localization system for an indoor rotary-wing MAV using blade mounted LEDs,” Sensor Review, Emerald, Vol.28, Issue 2, pp. 125-131, 2008.

5. D.Sun and J.L.Crassidis, "Observability analysis of six-degree-of freedom configuration determination using vector observations," Journal of Guidance, Control and Dynamics, Vol. 25, No. 6, pp. 1149-1157, Nov. 2002

6. R. Hartly, and A. Zisserman, Multiple View Geometry in Computer Vision, Cambridge University Press, 2003.

7. Strobl, K., Sepp, W., Fuchs, S., Paredes, C., and Arbter, K. Camera calibration toolbox for Matlab

8. M. K. Kalandros, L. Trailovic, L. Y. Pao and Y. Bar-Shalom, "Tutorial on Multisensor Management and Fusion Algorithms for Target Tracking," Proceeding of the 2004 American Control Conference, June 30 - July 2, 2004.

9. T. H. Chang, S. Gong and E. J. Ong, "Tracking multiple people under occlusion using multiple cameras," British Machine Vision Conference, Bristol, England, 2000.

10. S. Khan and M. Shah, "Tracking people in presence of occlusion," In Asian Conference on Computer Vision, 2000.

11. I.J. Cox, "A Review of Statistical Data Association Techniques for Motion Correspondence," International Journal of Computer Vision, Vol.10, pp. 53-66, 1993.

12. Brian L. Stevens and Frank L.Lewis, AIRCRAFT CONTROL AND SIMULATION, John Wiley \& Sons, Inc., 1992.

13. H. Oh, D. Won, S. Huh, B. Park, D. H. Shim and M. Tahk, "Indoor UAV Pose Estimation from Multi-Camera System Using EKF," 2nd International Symposium on Unmanned Aerial Vehicles, Reno, Nevada.

14. Dan Simon, Optimal State Estimation; H-inf and Nonlinear Approaches, John Wiley \& Sons, Inc, 2006.

15. H. L. Alexander, "State estimation for distributed systems with sensing delay," SPIE, Data Structures and Target Classification, Vol. 1470, pp. 103-111, 1991.

16. T. D. Larsen, N.A. Andersen and O. Ravn, "Incorporation of Time Delayed Measurements in a Discrete-time Kalman Filter," Proceedings on IEEE Conference on Decision and Control, 1998.

17. S. Thomopoulos and L. Zhang "Decentralized Filtering with Random Sampling and Delay," INFORMATION SCIENCES-Informatics and Computer Science: An International Journal 81(12), pp. 117-131, 1994.

18. S. Bouabdallah, P. Murrieri, and R. Siegwart, "Design and Control of an Indoor Micro Quadrotor," ICRA, New Orleans, April 2004.

19. G. F. Franklin, J. D. Powell and A. Emami-Naeini, Feedback Control of Dynamic Systems $4^{\text {th }}$, Prentice Hall. 